\title{
Intelligent Information Retrieval System Based on Muti-Agent Model
}

\author{
Zhaodan $\mathrm{Wu}^{1, \mathrm{a}}$, Changfeng $\mathrm{Shi}^{2, \mathrm{~b}}$, Yi $\mathrm{Lu}^{3, \mathrm{c}}$ \\ ${ }^{1,2,3}$ Business School, Hohai University, Nanjing, Jiangsu, China 211100 \\ ${ }^{1}$ Department of Earth and Environmental Engineering, Columbia University, New York City, NY, USA \\ 10027 \\ azhaodanwu@gmail.com, bshichangfeng007@163.com, '361168375@qq.com
}

Keywords: Information Retrieval, Intelligent Search, Agent, R-SCP analysis, MAS

\begin{abstract}
The intelligent information retrieval model discussed in this paper is constructed by multi-agent. Currently, BDI cognitive theory is accepted widely by the scholars of this field, but the related researches mainly focus on the theoretical derivation and presentation of symbols, lack of model facing practical application. In this article, a dynamic rule-based reasoning model is proposed. The model based on BDI theory is an expression of agent intelligence. The basic logical reasoning of the BDI theory is extended in this article. The author not only introduces several functions to study the dynamic changes of agents' mental state, but also give a detailed description of how to use the theory of production rules to express BDI-based reasoning. This article also studies and designs the agent communication mechanism in MAS. Finally, the intelligent information retrieval system is designed and implemented with the idea of AOP.
\end{abstract}

\section{Introduction}

With the continuous development of social information and Web technology, network information resources are becoming rich, but getting the information which meets our needs from the sea of information resources has become increasingly difficult. Although the use of major search engines and information retrieval tools owned by some sites can solve some problems. Search engines' sources of information are all Web resources of the Internet. Search engines do some processing to users' search text, such as word segmentation and fuzzy matching, etc. However, search engines do not crawl the web in real time. And search engines spider crawl rate is low. Most of search results are useless. It is hard to find useful information from these results. Most of the site's basic search functions are based on the search site database, so they are real-time and accurate information retrieval, however, they are isolated with each other and provide search functionality which is too simple.

The intelligent information retrieval model based on multi-agent imitates and synthesizes the features of search engine and information retrieval in the website. It realizes high recall-precision and high precision of the retrieval effectively, and has good flexibility and expansibility. It is convenient for users to configure and choose target source of retrieval.

Analysis, design and establishment of the system development, combined with MAS characteristics and AOP (Agent-oriented programming) thinking ${ }^{[1]}$ are discussed in this paper. First, analyzing and designing a single Agent model, and then analyze and design the intelligent information retrieval model as a whole (including the core part of the business and the auxiliary function part), the last part is the elaboration about multi-agent intelligent retrieval system's realization.

\section{The Agent General Structure Design}

Establish the Universal Agent model structure ${ }^{[2]}$, is the premise of design Multi-Agent Systems. All the specific Agent of Multi-Agent Systems Agent can be quickly designed and implemented on the base of the Universal Agent structure model. Through the above research, this paper designed a practical model of the Agent. The structure of the model not only can sense changes in the external 
environment to react accordingly, based on changes in their own state of mind to think smart manifestation.

\section{Agent Design, Human-computer Interaction}

Agent of human-computer interaction is rather a special Agent of all Agent system. Human-Computer Interaction Agent, to some extent, plays the role of the controller layer. Different from the other Agent, it inherits the Http Servlet parent class (based on the JAVA language), receives requests from the client browser. But at the same time, it has the Agent communication function which is used to communicate with other Agent in the system (as this article by using the multi-Agent architecture sake, and control of Agent to interact). Since this is not the user's interests and hobbies, the Human-Computer Interaction Agent system requirements are relatively loose. As shown in Figure 1, human-computer interaction Agent is to act as an important intermediary function. Equivalent to an adapter, coupled client browser and the actual multi-agent system.

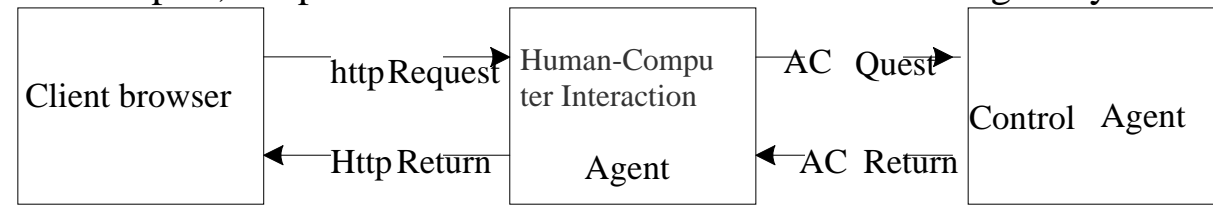

Fig. 1 the Main Flow of the Human-Computer Interaction Agent

\section{Control Agent Design}

Control Agent bear the role of the hub of the entire Multi-Agent Systems. It is the key to run the entire system effectively, to work together with Multi-Agent effectively. The Agent interaction must organized as an intermediate control by controlling the Agent. The following analysis based on the request of the Agent's three main business processes.

Firstly, Control Agent receive the request message from the Human-Computer Interaction Agent , shown in Figure 2, the message processor for processing the message, then Control Agent word processing Agent sends the need to test the connection reply message, if after a certain period of time $^{[3][4]}$, the reply has not received ,then enter the error handling process (the process flow does not go into detail), if you receive a reply, said both normal interaction, subsequent control Agent Agent sends a request segmentation without reply for word processing.

Secondly, word processing Agent to complete the processing of the segmentation will notify the control Agent (Figure 3). Control processing Agent receives message from word processing, message parsing process, resulting in the start search intent. Identify the current in all retrieved Agent Control Agent, then traverse each remove a search agent information on its reply to a message to send the connection test, then the retrieved Agent sends a request to retrieve information.

Finally, whenever received confirmation of completion messages from a specific retrieval Agent ,it is necessary to check receipt of all retrieved Agent (Figure 4). If not, continue to wait for the listener. After all the retrieved Agents play research function, the Human-Computer Interaction Agent test should be taken to make sense whether the communication is normal, then request the Human-Computer Interaction Agent access to search results ${ }^{[5]}$.

\section{Key Technologies to Achieve}

This section will focus on the realization of how multi-Agent intelligent information retrieval system. We make research on the key technologies as follows.

Listener Implementation Based on Socket. There are a lot of functional modules in agent. And degree of coupling between agents is well. So as an important of communication model, monitor needs to be separated from the main agent, to perform responsible for the task. The key modes are shown in Figure 5. All the retrieval agents in the Intelligent Information Retrieval System work at the same time. And all the specific retrieval process will use the thread mechanism. As the system 
processes the text which users enter . The retrieval agent received a lot of keywords. In order to improve the efficiency of the system, we will take consideration of multithreading, and for further, Agent will check the completion of all these sub-thread. The codes are shown in Figure 6. They describe in detail how thread works to in each Agent and how it make a judgment .

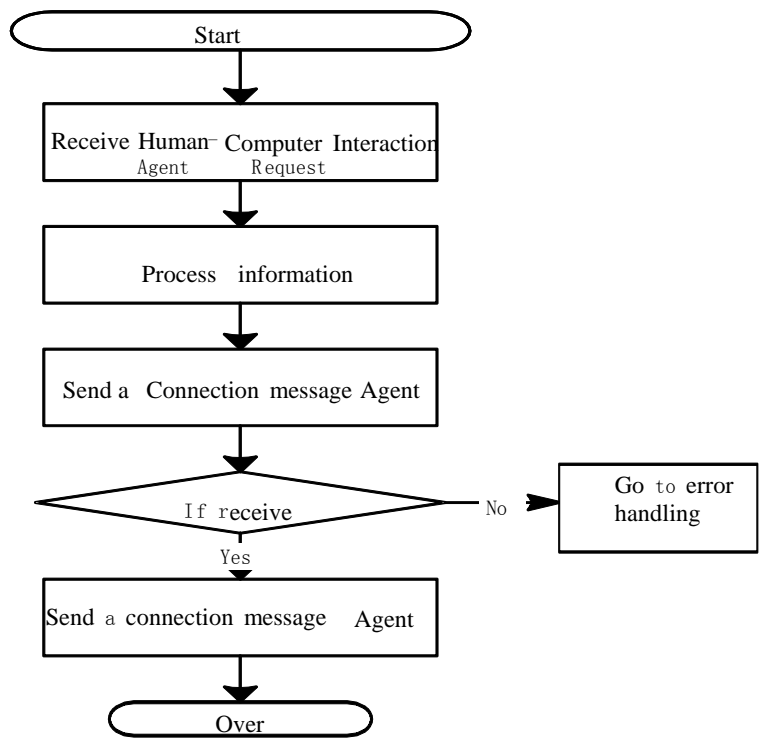

Fig.2 Control Agent Receives the Human-Computer Interaction Agent Request Process

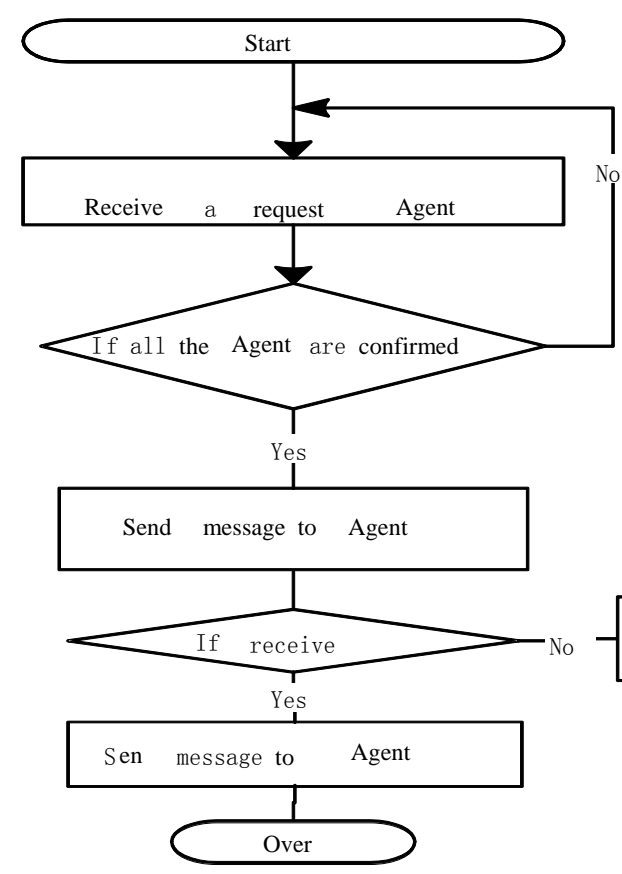

Go to the error handling process

Fig .4 Control Agent Receives to Retrieve Agent Request Process.

\section{Conclusions}

Based on the study of multi-agent communication mechanism, a single Agent general structural model is designed. The building of Multi-Agent intelligent information retrieval model primarily uses the thought of AOP, so it is necessary to design the general structure of the model of a single Agent firstly, the other specific Agent will reference the model structure and their own features, the 
core part of the whole intelligent retrieval system (search operations) based design and implementation-oriented these specific Agent.

$$
\begin{gathered}
\text { public class Monitor Thread extends Thread } \\
\text { • • / / Other variables }
\end{gathered}
$$

public final int socket port;//socket Port number

- • //Other methods public void run()

$$
\begin{gathered}
\{ \\
\text { Server Socket ss=null; } \\
\text { try }\{ \\
\text { ss = new ServerSocket(socket_port);//make a } \\
\text { new ServerSocket } \\
\text { while((s=ss.accept())!=null) }\{ \\
\text { Message mess=null;//information } \\
\text { Input Stream is=s.getInputStream(); } \\
\text { ObjectInputStream ois=new Object Input } \\
\text { Stream(is); } \\
\text { try }\{ \\
\text { mess=(Message)ois.readObject(); } \\
\text { \} catch (ClassNotFoundException e) }\{ \\
\text { e.printStackTrace(); } \\
\} \\
\text { • //process information } \\
\text { ois.close();//close the flue } \\
\text { s.close();//close Socket objects } \\
\} \\
\text { \} catch (IOException e) }\{ \\
\text { // TODO Auto-generated catch block } \\
\text { e.printStackTrace(); } \\
\} \\
\text { finally }\{ \\
\text { ss.close(); }
\end{gathered}
$$

Fig .5 Listener Implementation Based on Socket

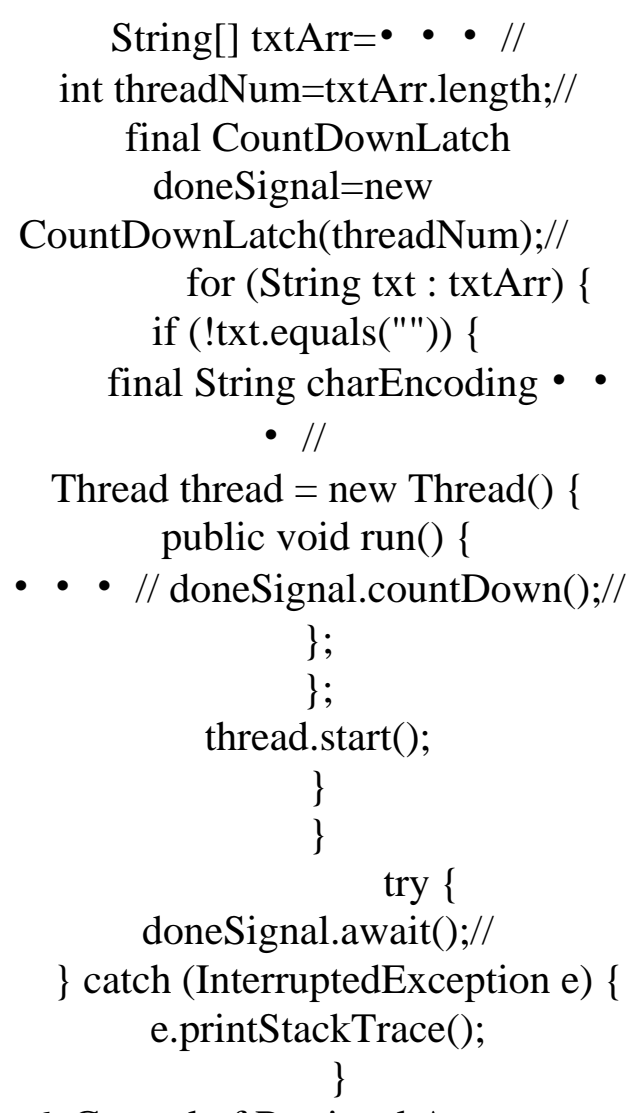

Fig .6 Control of Retrieval Agent

\section{Literature References}

[1] Singh M P. Multi-Agent System: A theoretical Framework for Intentions, Know-how, and Communications. New York: Springer-Verlag, 1994.

[2] S.Lawrence, C.L.Giles. Context and page analysis for improved web search[J].IEEE Internet Computing,1998.

[3] E.W.Selber.Towards Comprehensive Web Search[D].University of Washington,1999.

[4] S.Lawrence,C.L.Giles. Searching the World Wide Web[J]. Science, 1998.

[5] Bratman M E. Intentions,Plans, and Pratical Reason[D]. Cambridge, MA:Harvard University Press, 1987. 